\title{
New scheme for nonlinear Schrödinger equations with vari- able coefficients
}

\author{
Xiu-Ling Yin ${ }^{\mathrm{a}, *}$, Shu-Xia Kong ${ }^{\mathrm{a}}$, Yan-Qin Liu ${ }^{\mathrm{a}}$, Xiao-Tong Zheng ${ }^{\mathrm{b}}$ \\ a School of Mathematical Sciences, Dezhou University, Dezhou, China. \\ ${ }^{b}$ School of Statistics, Renmin University of China, Beijing, China.
}

\begin{abstract}
This paper proposes a numerical scheme for nonlinear Schrödinger equations with periodic variable coefficients and stochastic perturbation. The scheme is obtained by applying finite element method in spatial direction and finite difference scheme in temporal direction, respectively. The scheme is stable in the sense that it preserves discrete charge of the Schrödinger equations. The numerical examples verify the conservative property of the new scheme.
\end{abstract}

Keywords: Schrödinger equation, finite element method, finite difference scheme.

2010 MSC: 65M06, 65M12, 65Z05.

(C)2019 All rights reserved.

\section{Introduction}

Schrödinger-type equations are important mathematical physical models in plasma physics, nonlinear optics, engineering, celestial mechanics, Bose-Einstein condensations ([14]). With the idea of chirp in optical soliton communication, they are used to study the propagation of picosecond optical pulses in single-mode fiber. They can describe the state of motion of outer electrons and reflect the characteristic wave-particle duality. Schrödinger-type equations are mathematical models for energy transfer in a monolayer molecular aggregate in the presence of thermal fluctuations. With average field theorem, they are applied to analyze the property of macroscopic wave function of aggregate in Bose-Einstein condensation.

The research for deterministic Schrödinger-type equations is well done. There are many investigations for deterministic PDEs, such as exp-function method [8], homotopy perturbation method [23], variational iteration method [22], collocation scheme [16], finite difference method [9] and so on. The numerical analysis for deterministic Schrödinger-type equations, such as symplectic schemes [17] and multi-symplectic schemes [20], pseudospectral method [4], compact method [6], finite volume scheme [7], collocation method [24], and conservative scheme [12, 19], can be found in the references therein.

Stochastic Schrödinger-type equations become a hot research topic recently. Well-posedness results for nonlinear Schrödinger equation (NSE) with linear Wiener noise is proved in [1]. Multi-symplectic scheme is proposed for stochastic NSE in [10]. An exponential scheme is developed to simulate quantum

\footnotetext{
*Corresponding author

Email address: yinllmm@163.com (Xiu-Ling Yin)
}

doi: $10.22436 /$ jmcs.019.03.02

Received: 2017-08-04 Revised: 2019-04-13 Accepted: 2019-04-19 
observables of NSE [15]. A semi-discrete scheme is considered to NSE with power nonlinearity [5]. The Strang-type splitting scheme is proposed to NSE with multiplicative noise [13]. A $\theta$-scheme is analyzed for NSE with Stratonovich noise [3].

It is found that, finite element method is a kind of important numerical methods for NSE. A mixed finite-element method and the two-grid approach are applied to solve NSE [21]. Linearized CrankNicolson Galerkin method is studied for generalized NSE [18]. Continuous Galerkin methods on the space-time mesh are analyzed for NSE in [11]. Symplectic local discontinuous Galerkin method is proposed to NSE with multiplicative noise [2].

In this paper, we focus on the numerical investigations of conservative stochastic NSE. For conservative stochastic NSE, the reference of finite element method still lack. If we apply finite element method in space and finite difference scheme in time to conservative stochastic NSE, how does the obtained scheme behave? This motivates us to study the question in this paper. We try to observe the ability of our scheme in preserving conservative property of stochastic NSE.

In below we consider the initial-boundary problem of the following NSE with periodic variable coefficients and stochastic perturbation

$$
\left\{\begin{array}{l}
i u_{t}+\alpha(t) u_{x x}+\beta(t)|u|^{2} u=\epsilon u \circ \dot{x}, x \in I=[-a, a], t \in(0, T] \\
\mathfrak{u}(x, 0)=\varphi(x), u(a, t)=u(-a, t)=0, \quad x \in I, t \in[0, T]
\end{array}\right.
$$

where $i^{2}=-1, u_{t}$ and $u_{x x}$ mean the first order partial derivative of $u$ with respect to $t$ and the second order partial derivative of $u$ with respect to $x$, respectively. $\alpha(t)$ and $\beta(t)$ are real bounded functions, $\epsilon$ is a small real number, and $\circ$ means Stratnovich product. $\dot{\chi}$ is a real-valued white noise which is delta correlated in time, either smooth or delta correlated in space. $\varphi(x)$ is a differential function. For NSE, $\alpha(t) u_{x x}, \beta(t)|u|^{2} u$, and $\epsilon u \circ \dot{\chi}$ are dispersion term, nonlinear term, and potential disturbance term, respectively. The balance between the effect of these terms can maintain the spatial profile of soliton waves. If $\epsilon=0$, the system (1.1) is a deterministic system.

Proposition 1.1. Under the periodic boundary condition, the solution of (1.1) satisfies the charge conservation law:

$$
\mathcal{N}(\mathrm{t})=\int_{-\mathrm{a}}^{\mathrm{a}}|\mathrm{u}(\mathrm{x}, \mathrm{t})|^{2} \mathrm{~d} x=\mathcal{N}(0) .
$$

So we also say that the NSE system (1.1) is conservative.

Proof. By multiplying (1.1) by $\bar{u}$ and integrating it with respect to $x$, we obtain that

$$
i \int_{I} u_{t} \bar{u} d x+\alpha(t) \int_{I} u_{x x} \bar{u} d x+\beta(t) \int_{I}|u|^{4} d x=\epsilon \int_{I}|u|^{2} \circ \dot{x} d x .
$$

According to the boundary condition, we derive that

$$
i \int_{I} u_{t} \bar{u} d x-\alpha(t) \int_{I}\left|u_{x}\right|^{2} d x+\beta(t) \int_{I}|u|^{4} d x=\int_{I} \epsilon|u|^{2} \circ \dot{\chi} d x
$$

Taking the imaginary part of above equation yields that

$$
\int_{\mathrm{I}} u_{\mathrm{t}} \overline{\mathrm{u}}+\mathrm{u} \bar{u}_{\mathrm{t}} \mathrm{d} x=\frac{\mathrm{d}}{\mathrm{d} t} \int_{\mathrm{I}} u \bar{u} \mathrm{~d} x=0
$$

This implies the charge conservation law (1.2), which can be used to test the efficiency of numerical methods for the NSE system (1.1).

Denote $u=p+i q$. Then the NSE (1.1) can be written in the following form

$$
\left\{\begin{array}{l}
p_{t}+\alpha(t) q_{x x}+\beta(t)\left(p^{2}+q^{2}\right) q=\epsilon q \circ \dot{\chi} \\
-q_{t}+\alpha(t) p_{x x}+\beta(t)\left(p^{2}+q^{2}\right) p=\epsilon p \circ \dot{\chi}
\end{array}\right.
$$

\section{New numerical scheme}

In below, for simplicity, we apply the uniform mesh grids $\left\{x_{k}=-a+k h, t_{n}=n \tau\right\}$ with step-sizes $h=2 a / K$ and $\tau=T / M$. Numerical values of $u\left(x_{k}, t_{n}\right)$ are denoted by $u_{k}^{n}$. 
The standard Sobolev space is denoted by

$$
\mathrm{H}_{0}^{1}(\mathrm{I})=\left\{\psi(x) \mid \psi, \psi^{\prime} \in \mathrm{L}^{2}(\mathrm{I}), \psi(\mathrm{a})=\psi(-\mathrm{a})=0, x \in \mathrm{I}\right\} .
$$

For any $\psi \in \mathrm{H}_{0}^{1}(\mathrm{I})$, with the boundary condition and Green formula, we can derive the following weak equations of (1.3),

$$
\begin{array}{r}
\int_{I} p_{t} \psi d x-\alpha(t) \int_{I} q_{x} \psi_{x} d x+\beta(t) \int_{I}\left(p^{2}+q^{2}\right) q \psi d x=\epsilon \int_{I} q \psi \circ \dot{\chi} d x, \\
-\int_{I} q_{t} \psi d x-\alpha(t) \int_{I} p_{x} \psi_{x} d x+\beta(t) \int_{I}\left(p^{2}+q^{2}\right) p \psi d x=\epsilon \int_{I} p \psi \circ \dot{\chi} d x .
\end{array}
$$

Additionally, we denote the finite element space by $V_{h}$. Accordingly, the discrete form of the weak equations is: to find $p_{h}, q_{h} \in V_{h}$, such that for any $\psi_{h} \in V_{h}$, the following equations

$$
\begin{aligned}
& \int_{I}\left(p_{h}\right)_{t} \psi_{h} d x-\alpha(t) \int_{I}\left(q_{h}\right)_{x}\left(\psi_{h}\right)_{x} d x+\beta(t) \int_{I}\left(p_{h}^{2}+q_{h}^{2}\right) q_{h} \psi_{h} d x=a_{q^{\prime}}^{h} \\
- & \int_{I}\left(q_{h}\right)_{t} \psi_{h} d x-\alpha(t) \int_{I}\left(p_{h}\right)_{x}\left(\psi_{h}\right)_{x} d x+\beta(t) \int_{I}\left(p_{h}^{2}+q_{h}^{2}\right) p_{h} \psi_{h} d x=a_{p}^{h}
\end{aligned}
$$

are satisfied.

In below, we will solve the discrete weak equations (2.1) and (2.2). We choose the space of segmental continuous linear function as $V_{h}$. Then $V_{h}$ is the linear expansion space generated by $\phi_{1}, \phi_{2}, \ldots, \phi_{K+1}$, where

$$
\begin{aligned}
& \phi_{1}(x)=\left\{\begin{array}{ll}
\frac{x_{1}-x}{h}, & x \in\left(x_{0}, x_{1}\right], \\
0, & x \notin\left(x_{0}, x_{1}\right],
\end{array} \quad \phi_{K+1}(x)= \begin{cases}\frac{x-x_{K}}{h}, & x \in\left(x_{K}, x_{K+1}\right], \\
0, & x \notin\left(x_{K}, x_{K+1}\right],\end{cases} \right. \\
& \phi_{k}(x)=\left\{\begin{array}{ll}
\frac{x-x_{k-1}}{h}, & x \in\left(x_{k-1}, x_{k}\right], \\
\frac{x_{k+1}-x}{h}, & x \in\left(x_{k}, x_{k+1}\right], \\
0, & x \notin\left(x_{k-1}, x_{k+1}\right],
\end{array} \quad k=2, \ldots, k .\right.
\end{aligned}
$$

Suppose that

$$
p_{h}=\sum_{k=1}^{K+1} a_{k}(t) \phi_{k}(x), q_{h}=\sum_{k=1}^{K+1} b_{k}(t) \phi_{k}(x) .
$$

Replacing $\psi_{\mathrm{h}}$ by $\phi_{\mathrm{j}}$ and inserting (2.3) into (2.1) and (2.2) yield that

$$
\begin{aligned}
& A P^{\prime}-[\alpha(t) B-\beta(t) D(P, Q)+\epsilon C] Q=0, \\
& A Q^{\prime}+[\alpha(t) B-\beta(t) D(P, Q)+\epsilon C] P=0,
\end{aligned}
$$

where $P=\left(a_{1}, a_{2}, \ldots, a_{K+1}\right)^{T}, Q=\left(b_{1}, b_{2}, \ldots, b_{K+1}\right)^{T}, A=\left(a_{k j}\right), B=\left(b_{k j}\right), C=\left(c_{k j}\right)$, and $D(P, Q)=$ $\left(d_{k j}\right)$ with the following notations

$$
\begin{aligned}
& \mathrm{a}_{\mathrm{kj}}=\int_{\mathrm{I}} \phi_{\mathrm{k}} \phi_{\mathrm{j}} \mathrm{d} x, \mathrm{~b}_{\mathrm{kj}}=\int_{\mathrm{I}} \phi_{\mathrm{k}}^{\prime} \phi_{j}^{\prime} \mathrm{d} x, \mathrm{c}_{\mathrm{kj}}=\int_{\mathrm{I}} \phi_{\mathrm{k}} \phi_{j} \circ \dot{\chi} \mathrm{d} x, \\
& \mathrm{~d}_{\mathrm{kj}}=\int_{\mathrm{I}}\left(\left|\sum_{\mathrm{m}=1}^{\mathrm{K}+1} \mathrm{a}_{\mathrm{m}}(\mathrm{t}) \phi_{\mathrm{m}}\right|^{2}+\left|\sum_{\mathrm{m}=1}^{\mathrm{K}+1} \mathrm{~b}_{\mathrm{m}}(\mathrm{t}) \phi_{\mathrm{m}}\right|^{2}\right) \phi_{\mathrm{k}} \phi_{j} \mathrm{~d} x .
\end{aligned}
$$

Then we apply the following finite difference scheme to (2.4)-(2.5) and get the new scheme for the NSE system (1.3),

$$
\begin{aligned}
& A \frac{P^{n+1}-P^{n}}{\tau}-\left[\alpha_{n} B-\beta_{n} D\left(P^{n}, Q^{n}\right)+\epsilon C^{n}\right] Q^{n+\frac{1}{2}}=0, \\
& A \frac{Q^{n+1}-Q^{n}}{\tau}+\left[\alpha_{n} B-\beta_{n} D\left(P^{n}, Q^{n}\right)+\epsilon C^{n}\right] P^{n+\frac{1}{2}}=0,
\end{aligned}
$$

where $\alpha_{n}=\alpha\left(t_{n}\right), \beta_{n}=\beta\left(t_{n}\right), P^{n+1 / 2}=\left(P^{n+1}+P^{n}\right) / 2, Q^{n+1 / 2}=\left(Q^{n+1}+Q^{n}\right) / 2$. The elements in 
$\mathrm{D}(\mathrm{P}, \mathrm{Q}), \mathrm{C}$ are substituted by the numerical solutions to get $\mathrm{D}\left(\mathrm{P}^{\mathrm{n}}, \mathrm{Q}^{\mathrm{n}}\right), \mathrm{C}^{\mathrm{n}}$.

Theorem 2.1. Denote $\mathrm{U}^{\mathrm{n}}=\mathrm{P}^{\mathrm{n}}+\mathrm{i} \mathrm{Q}^{\mathrm{n}}$. Let $\left\|\mathrm{U}^{\mathrm{n}}\right\|^{2}=\mathrm{h}\left|\mathrm{U}^{\mathrm{n}}\right|^{2}=\mathrm{h} \sum \mathrm{U}_{\mathrm{k}}^{\mathrm{n}} \overline{\mathrm{U}_{\mathrm{k}}^{\mathrm{n}}}$. Then $\left\|\mathrm{U}^{\mathrm{n}}\right\|^{2}$ is the discrete charge invariant of the scheme (2.6)-(2.7), which implies the discrete charge conservation law of (1.1). So in this sense, we also say that the scheme (2.6)-(2.7) behaves conservatively and stably.

Proof. The scheme (2.6)-(2.7) is equivalent to

$$
A \frac{U^{n+1}-U^{n}}{\tau}+i\left[\alpha_{n} B-\beta_{n} D\left(P^{n}, Q^{n}\right)+\epsilon C^{n}\right] U^{n+\frac{1}{2}}=0,
$$

where $U^{n+1 / 2}=\left(U^{n+1}+U^{n}\right) / 2$. This is a finite difference scheme to the NSE (1.1). By multiplying (2.8) with $\overline{\mathrm{U}^{\mathrm{n}+\frac{1}{2}}}$, we obtain that

$$
A \frac{\left|U^{n+1}\right|^{2}-\left|U^{n}\right|^{2}+U^{n+1} \overline{U^{n}}-\overline{U^{n+1}} U^{n}}{2 \tau}+i\left[\alpha_{n} B-\beta_{n} D\left(P^{n}, Q^{n}\right)+\epsilon C^{n}\right]\left|U^{n+\frac{1}{2}}\right|^{2}=0 .
$$

Considering the real symmetry and invertibility of $A$, the real part of above equation (2.9) implies that

$$
\left\|\mathrm{U}^{\mathrm{n}}\right\|_{2}=\left\|\mathrm{U}^{\mathrm{n}+1}\right\|_{2} \text {. }
$$

\section{Numerical results}

We apply above scheme (2.6)-(2.8) to solve three NSEs with periodic boundary condition and test the conservative property of the scheme. We consider $\left\|U^{n}\right\|$ as the discrete charge of $\mathcal{N}\left(t_{n}\right)$. The numerical residuals of $\mathcal{N}(t)$ is measured by the error $\left\|\mathrm{U}^{\mathrm{n}}\right\|-\left\|\mathrm{U}^{0}\right\|$.

Example 3.1. First we consider the following NSE with constant coefficients

$$
\left\{\begin{array}{l}
i u_{t}+u_{x x}+|u|^{2} u=\epsilon u \circ \dot{\chi}, \quad x \in I=[-5,5], t>0, \\
u(x, 0)=\frac{\sqrt{2}}{2} \exp \left(i \frac{x}{2}\right) \operatorname{sech}\left(\frac{x}{2}\right), u(5, t)=u(-5, t), \quad x \in I, t>0 .
\end{array}\right.
$$

The step-sizes we used are $h=1 / 8$ and $\tau=1 / 100$. In Figure 1, we plot the discrete charge (left) and its residuals (right) for numerical solutions of (3.1) with $\epsilon=0.4$, respectively. The figure verifies that our scheme preserves the discrete charge conservation law of (3.1) approximately. Numerical results are similar to other step-sizes $h, \tau$ and other perturbation size $\epsilon$.
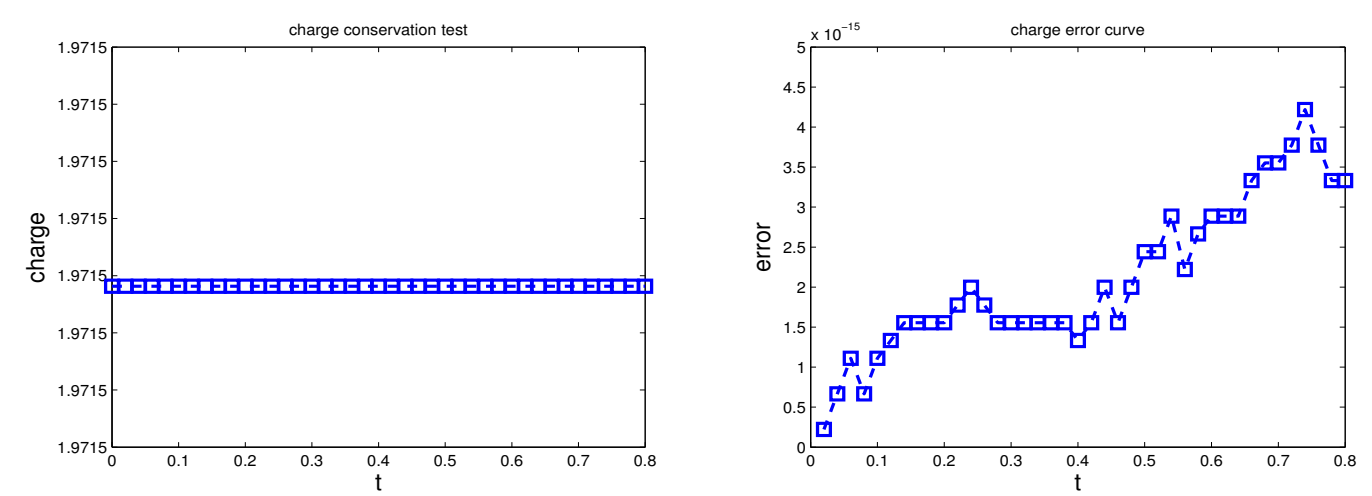

Figure 1: Discrete charge (left) and its residuals (right) for numerical solutions of the NSE (3.1). 
Example 3.2. Second, we consider the following NSE with variable coefficients

$$
\left\{\begin{array}{l}
i u_{t}+\frac{1}{2} \cos (t) u_{x x}+\frac{\cos (t)}{\sin (t)+3}|u|^{2} u=\epsilon u \circ \dot{\chi}, \quad x \in I=[-5,5], t>0, \\
u(x, 0)=\frac{\sqrt{3}}{3} \exp \left(\frac{i\left(x^{2}-1\right)}{6}\right) \operatorname{sech}\left(\frac{x}{3}\right), u(5, t)=u(-5, t), \quad x \in I, t>0 .
\end{array}\right.
$$

The step-sizes we applied are $h=1 / 8$ and $\tau=1 / 100$. In Figure 2, we depict the discrete charge (left) and its residuals (right) for numerical solutions of (3.2) with $\epsilon=0.5$, respectively. The figure shows that the method also preserves the discrete charge invariant of (3.2) approximately. To other step-sizes $h, \tau$ and other perturbation size $\epsilon$, we obtain similar numerical results.
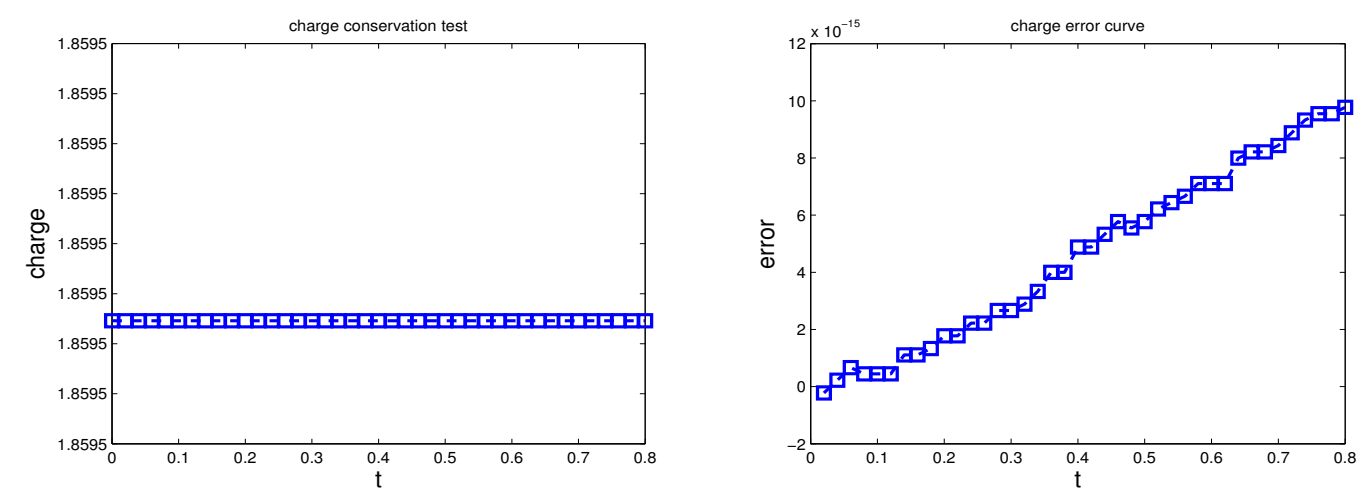

Figure 2: Discrete charge (left) and its residuals (right) for numerical solutions of the NSE (3.2).

Example 3.3. Third, we consider the following NSE with variable coefficients

$$
\left\{\begin{array}{l}
i u_{t}+\frac{1}{2}[\cos (t)+\sqrt{2} \cos (\sqrt{2} t)] u_{x x}+\frac{\cos (t)+\sqrt{2} \cos (\sqrt{2} t)}{\sin (t)+\sin (\sqrt{2} t)+5}|u|^{2} u=\epsilon u \circ \dot{\chi}, x \in I=[-5,5], t>0 \\
u(x, 0)=\frac{\sqrt{5}}{5} \exp \left(\frac{i\left(x^{2}-1\right)}{10}\right) \operatorname{sech}\left(\frac{x}{5}\right), u(5, t)=u(-5, t), \quad x \in I, t>0 .
\end{array}\right.
$$

The step-sizes we used are $h=1 / 8$ and $\tau=1 / 100$. In Figure 3, we plot the discrete charge (left) and its residuals (right) for numerical solutions of (3.3) with $\epsilon=0.6$, respectively. The figure tells us that the method preserves the discrete charge conservative property of (3.3) approximately. It follows analogously for other step-sizes $h, \tau$ and other perturbation size $\epsilon$.
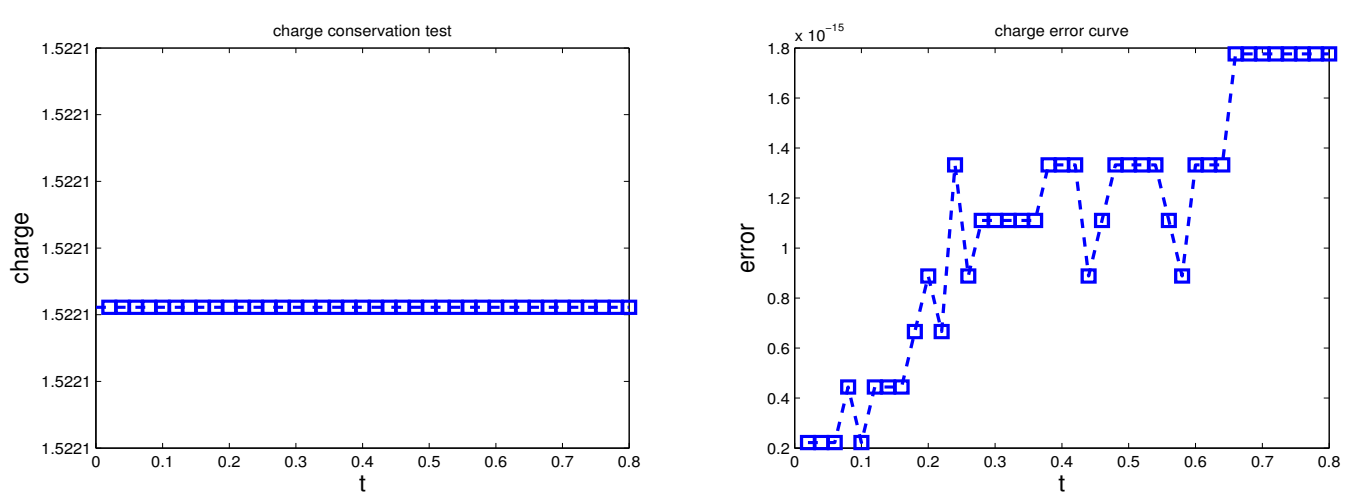

Figure 3: Discrete charge (left) and its residuals (right) for numerical solutions of the NSE (3.3).

In Figure 4, we plot the three-dimensional graph for imaginary parts of numerical solutions for Example 3.1 (left), Example 3.2 (middle), and Example 3.3 (right), respectively. Although the profile of soliton waves is maintained, the small noise produces stochastic fluctuations on the waves. 

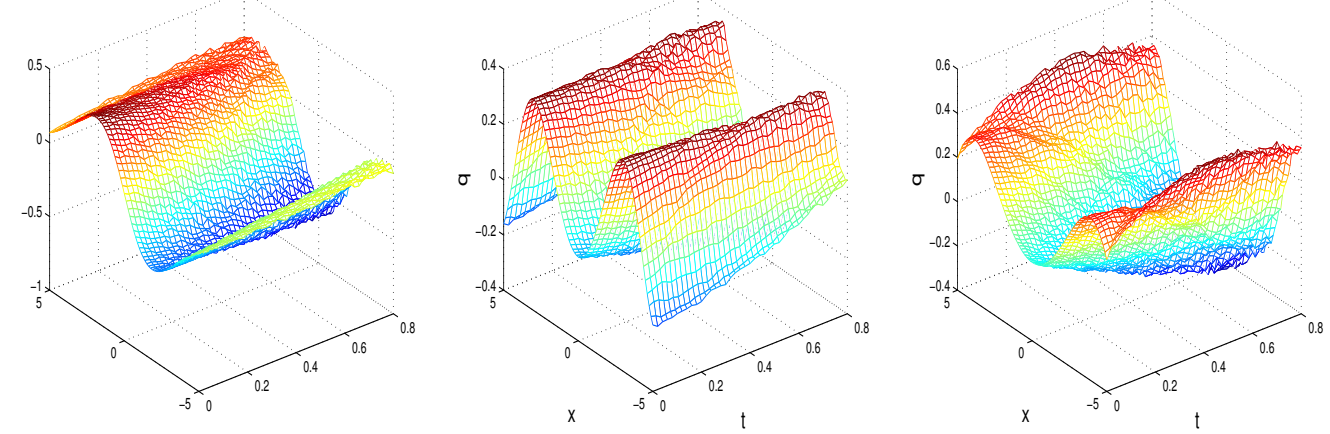

Figure 4: Imaginary parts graph for numerical solutions of the NSEs.

\section{Conclusion}

We combine finite element method and finite difference scheme to obtain a new scheme to NSEs. The stable scheme has conservative discrete charge for NSEs. We also investigate the scheme for three NSE examples with periodic variable coefficients and stochastic perturbation. Further discussion and research about the numerical analysis of stochastic NSEs will be followed.

\section{Acknowledgment}

This work is supported by National Natural Science Foundation of China (Nos. 11801060, 11501082), Natural Science Foundation of Shandong Province (Nos. ZR2018LA001, ZR2017LA012, ZR2017MA050, ZR2018LA003), and Science and Technology Program of Colleges and Universities in Shandong (No. J17KA156). The authors appreciate valuable comments of the reviewers.

\section{References}

[1] V. Barbu, M. Röckner, D. Zhang, Stochastic nonlinear Schrödinger equations with linear multiplicative noise: rescaling approach, J. Nonlinear Sci., 24 (2014), 383-409. 1

[2] C. C. Chen, J. L. Hong, L. H. Ji, A symplectic local discontinuous Galerkin method for stochastic Schrödinger equation, arXiv, 2014 (2014), 19 pages. 1

[3] C. C. Chen, J. L. Hong, A. Prohl, Convergence of a $\theta$-scheme to solve the stochastic nonlinear Schrödinger equation with Stratonovich noise, Stochastic Part. Diff. Equ. Anal. Comput., 4 (2016), 274-318. 1

[4] J.-B. Chen, M.-Z. Qin, Multi-symplectic Fourier pseudospectral method for the nonlinear Schrödinger equation, Electron. Trans. Numer. Anal., 12 (2001), 193-204. 1

[5] A. de Bouard, A. Debussche, Weak and strong order of convergence of a semidiscrete scheme for the stochastic nonlinear Schrödinger equation, Appl. Math. Optim., 54 (2006), 369-399. 1

[6] M. Dehghan, A. Taleei, A compact split-step finite difference method for solving the nonlinear Schrödinger equation with constant and variable Coefficients, Comput. Phys. Commun., 181 (2010), 43-51. 1

[7] W. Gao, H. Li, Y. Liu, X. X. Wei, A Padé compact high-order finite volume scheme for nonlinear Schrödinger equations, Appl. Numer. Math., 85 (2014), 115-127. 1

[8] J.-H. He, M. A. Abdou, New periodic solutions for nonlinear evolution equations using Exp-function method, Chaos Soliton Fractals, 34 (2007), 1421-1429. 1

[9] B. İnan, A. R. Bahadir, Numerical solutions of MRLW equation by a fully implicit finite-difference scheme, J. Math. Computer Sci., 15 (2015), 228-239. 1

[10] S. S. Jiang, L. J. Wang, J. L. Hong, Stochastic multi-symplectic integrator for stochastic nonlinear Schrödinger equation, Commun. Comput. Phys., 14 (2013), 393-411. 1

[11] O. Karakashian, C. Makridakis, A space-time finite element method for the nonlinear Schrödinger equation: the continuous Galerkin method, SIAM J. Numer. Anal., 36 (2006), 1779-1807. 1

[12] L. H. Kong, P. Wei, Y. Q. Hong, P. Zhang, P. Wang, Efficient energy-preserving scheme of the three-coupled nonlinear Schrödinger equation, Math. Meth. Appl. Sci., 2019 (2019), 14 pages. 1

[13] J. Liu, Order of convergence of splitting schemes for both deterministic and stochastic nonlinear Schrödinger equations, SIAM J. Numer. Anal., 51 (2013), 1911-1932. 1 
[14] G. N. Milstein, M. V. Tretyakov, Stochastic Numerics for Mathematical Physics, Springer-Verlag, Berlin, (2004). 1

[15] C. M. Mora, Numerical solution of conservative finite-dimensional stochastic Schrödinger equations, Ann. Appl. Probab., 15 (2005), 2144-2171. 1

[16] M. Sarboland, A. Aminataei, Integrated high accuracy multiquadric quasi-interpolation scheme for solving the nonlinear Klein-Gordon equation, J. Math. Computer Sci., 14 (2015), 258-273. 1

[17] Y. F. Tang, J. W. Cao, X. T. Liu, Y. C. Sun, Symplectic methods for the Ablowitz-Ladik discrete nonlinear Schrödinger equation, J. Phys. A, 40 (2007), 2425-2437. 1

[18] J. L. Wang, A new error analysis of Crank-Nicolson Galerkin FEMs for a generalized nonlinear Schrödinger equation, J. Sci. Comput., 60 (2014), 390-407. 1

[19] T. C. Wang, B. L. Guo, Q. B. Xu, Fourth-order compact and energy conservative difference schemes for the nonlinear Schrödinger equation in two dimensions, J. Comput. Phys., 243 (2013), 382-399. 1

[20] Y. S. Wang, Q. H. Li, Y. Z. Song, General: two new simple multi-Symplectic schemes for the nonlinear Schrödinger equation, Chinese Phys. Lett., 25 (2008), 1538-1540. 1

[21] L. Wu, Two-grid mixed finite-element methods for nonlinear Schrödinger equations, Numer. Methods Partial Differential Equations, 28 (2012), 63-73. 1

[22] X.-J. Yang, D. Baleanu, Y. Khan, S. T. Mohyud-Din, Local fractional variational iteration method for diffusion and wave equations on Cantor sets, Romanian J. Phys., 59 (2014), 36-48. 1

[23] Y. Zhang, C. Cattani, X.-J. Yang, Local fractional homotopy perturbation method for solving non-homogeneous heat conduction equations in fractal domains, Entropy, 17 (2015), 6753-6764. 1

[24] H. J. Zhu, Y. M. Chen, S. H. Song, H. Y. Hu, Symplectic and multi-symplectic wavelet collocation methods for twodimensional Schrödinger equations, Appl. Numer. Math., 61 (2011), 308-321. 1 